\title{
Effects of chronic lead ingestion in adult rats
}

\author{
THOMAS LANTHORN and ROBERT L. ISAACSON \\ University of Florida, Gainesville, Florida 32611
}

\begin{abstract}
Adult rats were given lead $(0.27 \% \mathrm{~Pb})$ in their drinking water and compared with pair-fed controls on several behavior problems: spontaneous alternation and two learning problems with three reversals of each. The lead-treated animals had reduced rates of spontaneous alternation and difficulty in changing behavior when the cues signaling reward and nonreward were reversed. In addition, animals without much handling showed exaggerated responsiveness. These changes are similar, but less extreme, than those seen in neonates treated with lead. The development of a peripheral absorption barrier may account for the reduced sensitivity of adults to lead poisoning relative to similar poisoning with younger subjects.
\end{abstract}

Adverse behavioral effects have been reported following the ingestion of lead in both humans and animals. Behavioral effects of chronic administration of lead are most pronounced in the neonatal or very young animals. Brown (1975), for example, has reported evidence of an impairment of performance in a T-maze light-dark discrimination problem by young adult rats (8 weeks old) when lead was administered through the mother's milk on Postnatal Days 1 through 10. The impairment was not found if the lead was given on Postnatal Days 11 through 20.

The insensitivity of older animals to lead ingestion may be due to the maturation of absorption mechanisms, e.g., intestinal and "blood-brain" uptake processes. Brown (1975) found that blood levels of lead in rat pups increased when the nursing dams were fed lead on Postnatal Days 1 through 10, but blood levels were not changed if pups were nursed by dams ingesting lead on Postnatal Days 11 through 20.

While young animals may be more sensitive to lead poisoning, behavioral changes may occur in adult animals. It is possible that the difficulty in finding behavioral consequences of lead ingestion in adult animals may be due to the selection of behavioral tasks which are not the most sensitive to lead-induced damage.

In the brain, lead has been found to be concentrated in the hippocampus of normal and poisoned animals, primarily in the hilus of the dentate gyrus (Danscher, 1976; Fjerdingstad et al., 1974; Niklowitz, 1973). It may be that an excessive accumulation of lead would preclude the normal operation of hippocampal formation. To test the possibility that aberrant hippocampal function may underlie some of the consequences of lead poisoning, we undertook the study

This research was supported by Grant NIH RR 07-021-11 to the University of Florida. of adult animals ingesting lead in their drinking water on tasks that have been shown to be sensitive to hippocampal damage.

The animals were trained in a light-dark discrimination problem in a $\mathrm{T}$-maze, since similar problems have been used by other investigators as a test for the effects of lead exposure early in life. Recently, procedures have been developed for detailed analysis of several aspects of performance in this task by Olton and Samuelson (1974) that might be useful in detection of relatively small, but consistent, behavioral changes. In a second behavioral task, rats were trained to go to a particular spatial position. Animals with bilateral hippocampal damage are impaired in reversal performance on this task (Kimble \& Kimble, 1965). In addition, the animals were tested for spontaneous alternation in a T-maze. This task is reliable as an indicator of hippocampal destruction (Douglas, 1966).

It became evident early in the course of the experiment that the lead-treated animals were more reactive to handling. Therefore, a test of reactivity was included in this study. A new group of experimentally naive animals was also subjected to the administration of lead and tested for reactivity to handling.

\section{METHOD}

\section{Subjects}

The subjects for the maze study were 10 male Long-Evans hooded rats weighing $185-210 \mathrm{~g}$ at the beginning of the study. An additional 7 male rats, of approximately the same age and weight as the first 10 , were tested only on the reactivity tests. All subjects were individually housed with food and water given in the home cage, and maintained on a 12-h-12-h light/dark cycle.

\section{Procedures}

Lead. Six rats were randomly assigned to receive lead acetate in their drinking water. Like their solid food, this was supplied ad lib, except during training on the T-maze learning tasks. During $\mathrm{T}$-maze training, water containing $0.5 \%$ lead acetate $(0.27 \% \mathrm{~Pb})$ was supplied for $1 / 2 \mathrm{~h}$ per day in the home cage. Four other 
rats received $0.5 \%$ sodium acetate in their drinking water. Of the seven additional rats used for reactivity tests, four received lead acetate and three sodium acetate in their water. This was supplied for 2 weeks prior to testing.

Pair feeding. Animals that ingest moderate-to-large doses of lead become anorexic. The reduction in normal weight gain could be a confounding factor in behavioral changes. Control rats were paired with lead-treated rats having the most similar original weights. All lead-treated rats were given $50 \mathrm{~g}$ of Purina Rat Chow each day, which was more than any rat ate in 1 day. The next day the amount of food consumed was recorded and that amount was given to the paired-control animal.

Spontaneous alternation. Lead was administered for 2 weeks before behavioral testing was begun. Spontaneous alternation was then recorded for 5 days (two trials/day) in a T-maze. Percent alternation was then determined according to the formula of Douglas (1966).

Light discrimination. Two days after the end of the alternation testing, subjects were put on a $231 / 2-h$ water-deprivation schedule. The next day, the light-discrimination problem was begun. The maze and running procedures were the same as described by Olton and Samuelson (1974). Instead of photoelectric cells, the experimenter operated a switch connected to equipment that recorded the "run time" (time to traverse the initial stem of the maze), "choice time" (time to choose and enter an arm), and "goal time" (time from choice point to reach water goal). Ten trials were given each day, and the subjects were run in two groups of five each to approximate the intertrial interval used in the Olton and Samuelson study. Criterion performance was defined as at least $90 \%$ correct responses on 2 successive days, or 25 days if criterion performance had not been reached before then. Subjects were originally trained to go to the lighted goal area. Immediately after achieving criterion performance, the responses to the other goal area were rewarded and reward omitted from the original area. A total of three reversals were run unless a subject did not meet criterion on an earlier reversal problem. The position of the light was randomly varied in accordance with the schedule used by Olton and Samuelson.

Spatial discrimination. Immediately after completing the lightdiscrimination task, the subjects were trained on a positiondiscrimination problem in the same $\mathrm{T}$-maze with the light continued as an irrelevant cue. The position of the light was determined by the schedule used by Olton and Samuelson, but a correct response consisted of going to one of the two goal areas regardless of the light. A $90 \%$ correct response criterion was again used, but only 12 days ( 120 trials) was the maximum training allowed. After meeting criterion on the original position task (entering the right arm was rewarded), three reversals of the rewarded position were carried out.

Reactivity tests. After the spatial discrimination was completed, the subjects were left unhandled for 2 weeks. During that time, lead or sodium acetate was introduced in the drinking water of seven additional rats. Reactivity tests, as used by King (1959), were carried out for 8 successive days. These six tests were: (1) reaction to a pencil held directly in front of the rat, (2) reaction to light tapping on the back with the pencil, (3) resistance to capture, (4) resistance to handling, (5) vocalization to capture and handling, and (6) urination and defecation to capture and handling.

These tests were carried out in the animal's home cage in the colony room. They were begun at 7:30 a.m., at the beginning of the light cycle.

Statistical tests. Differences between groups were analyzed by $t$ tests, except for the reactivity testing which used a two-way analysis of variance with repeated measures followed by individual $t$ tests.

\section{Results}

Rats ingesting lead had reduced rates of spontaneous alternation. The mean rate of alternation in pair-fed control rats was $73.4 \%$ over all test days. One lead-treated subject was eliminated from the analysis because it refused to make a choice in the majority of the trials. The alternation rate in the remaining lead-treated rats was $62.0 \%$. The difference between these rates was significant $(p<.05)$.

Statistical analyses indicated a difference in the mean number of trials to criterion between the leadtreated animals and control animals on only one of the learning tasks. On the first spatial position task, which immediately followed the last light-discrimination test, rats receiving lead took significantly $(p<.05)$ more trials to reach criterion. No significant differences were found in the run time, choice time or goal time. No control subject failed to reach criterion on any of the light- or spatial-discrimination problems. Three of the lead-treated animals failed at least one of the discrimination problems or a reversal. One of these animals failed one reversal during the light-discrimination problem and two reversals of the spatial-discrimination problem. One lead-treated animal failed to acquire the spatial discrimination task.

The results of the reactivity tests are shown in Figure 1. A two-way analysis of variance with repeated measures run on the results of 8 days of reactivity testing, indicated that the naive rats receiving lead acetate were more reactive than all control

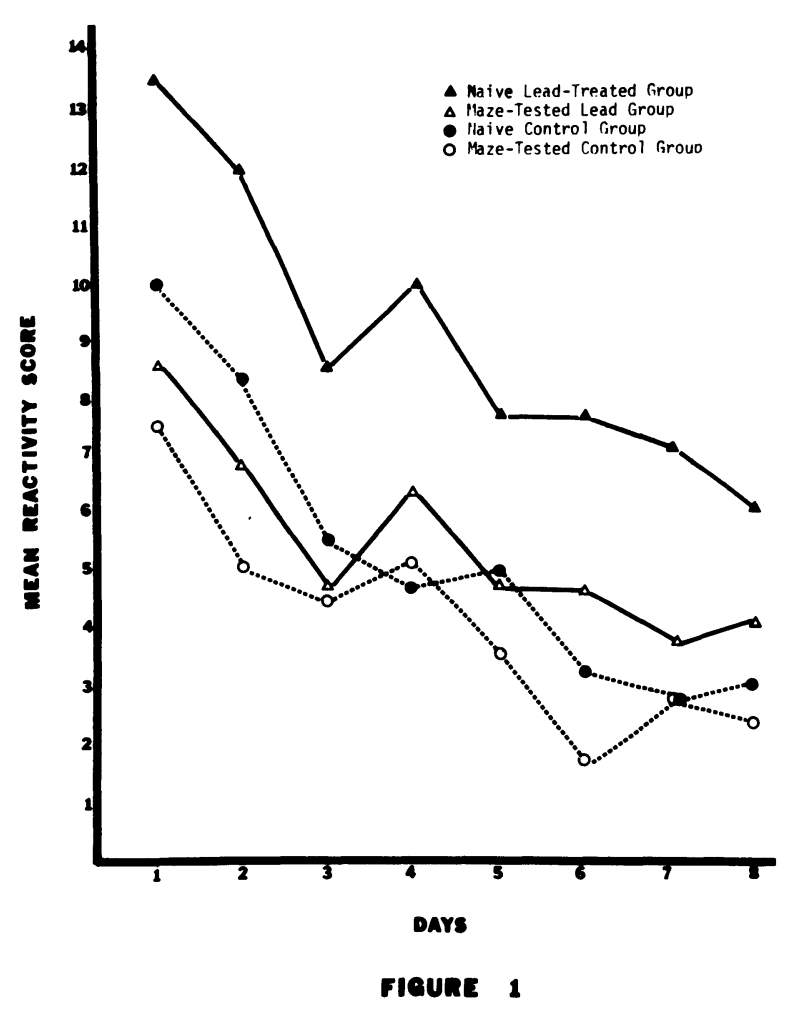

Figure 1. The mean reactivity scores of the lead-treated and control subjects. 
animals $(p<.01)$. Maze-tested controls did not differ from naive controls or from maze-tested leadtreated animals. The maze-tested lead-treated animals were less reactive than the naive lead-treated rats $(\mathrm{p}<.05)$.

\section{Discussion}

These results indicate that behavioral changes induced by lead ingestion in adult rats are like many of those induced in neonatal rats, although less severe. For example, the heightened reactivity found in the unhandled lead-treated animals, such as struggling and biting during capture and being held, is similar to the hyperactivity and aggressiveness seen in neonatally treated animals.

If lead treatment in adulthood produces learning impairments, simple T-maze problems do not seem to be very sensitive instruments for their measurement. In neonatally treated animals, an impairment was found by Brown (1975) on a successive discrimination task using light as the relevant cue. However, rats given a single does of lead during development showed no impairment on a simple spatial position discrimination task in a water-filled T-maze (Brown, 1971). In the present experiments with adults, leadinduced behavioral impairments were found only when the basis for a correct response was changed from light to the spatial location. Individual failures to reach criterion by lead-treated subjects did occur. Interestingly, all these failures occurred because of the persistent adoption of a left-turn habit.

The results also show that spontaneous alternation, a test sensitive to hippocampal damage, demonstrated a difference between lead-treated and pair-fed control rats. The failure to alternate by lead-treated rats was associated with a strong tendency to turn left. A recent report (Kostas et al., 1976) showed reduced spontaneous alternation following lead exposure during the neonatal period. Thus, it seems that spontaneous alternation may be a task that is sensitive to lead-induced behavioral changes. An apparent discrepancy in this study is the relatively low rate of alternation (73.4\%) exhibited by pair-fed controls. The reported value for normal rats is about $82 \%$. Douglas (1966) has shown that the major cues in spontaneous alternation are the rat's own odor trail and spatial orientation of the maze. The procedure used here eliminated the possibility of an animal following its own odor trail and left spatial location as the sole determinant of spontaneous alternation. Douglas reported that spatial orientation alone induced alternation of $75 \%$. The alternation rate reported here is close to that value. It is also possible that the pair-feeding procedure, which meant some food deprivation, may have reduced the alternation rate of the control animals.

While the effects of the oral ingestion of lead is more prominent in neonates, adverse effects can be found in mature animals following chronic lead ingestion.

\section{REFERENCES}

Brown, D. R. Neonatal lead exposure in the rat: Decreased learning as a function of age and blood lead concentrations. Toxicology and Applied Pharmacology, 1975, 32, 628-637.

Brown, S., Gragann, N., \& Vogel, W. H. Effects of lead acetate on learning and memory in rats. Archives of Environmental Health, 1971, 22, 370-372.

Danscher, G., Fjerdingstad, E. J., Fuerdingstad, E., \& Fredens, K. Heavy metal content in subdivisions of the rat hippocampus (zinc, lead, copper). Brain Research, 1976, 112, 442-446.

Douglas, R. J. Cues for spontaneous alternation. Journal of Comparative and Physiological Psychology, 1966, 62, 171-183.

Fuerdingstad, E. J., Danscher, G., \& Fuerdingstad, E. Hippocampus: Selective concentration of lead in the normal rat brain. Brain Research, 1974, 80, 350-354.

Kimble, D. P., \& Kimble, R. J. Hippocampectomy and response perseveration in the rat. Journal of Comparative and Physiological Psychology, 1965, 60, 474-476.

KING, F. A. Relationship of the "septal syndrome" to genetic differences in emotionality in the rat. Psychological Reports, 1959, 5, 11-17.

Kostas, J., McFarland, D. J., \& Drew, W. G. Lead-induced hyperactivity: Chronic exposure during the neonatal period in the rat. Pharmacology, 1976, 14, 435-442.

Niklowitz, W. J., \& YeAger, D. W. Interference of $\mathrm{Pb}$ with essential brain tissue $\mathrm{Cu}, \mathrm{Fe}$, and $\mathrm{Zn}$ as main determinant in experimental tetraethyllead encephalopathy. Life Sciences, 1973, 13, 897-905.

Olton, D. S., \& Samuelson, R. Decision making in the rat: Response-choice and response-time measures of discrimination reversal learning. Journal of Comparative and Physiological Psychology, 1974, 87, 1134-1147.

(Received for publication September 1, 1977; revision accepted November $30,1977$. ) 